\title{
Prevalence of poor psychiatric status and sleep quality among frontline healthcare workers during and after the COVID-19 outbreak: a longitudinal study
}

Yifang Zhou ${ }^{1,2}$, Hailong Ding ${ }^{3}$, Yifan Zhang ${ }^{1}$, Baoyan Zhang ${ }^{1}$, Yingrui Guo ${ }^{1}$, Teris Cheung ${ }^{4}$, Brian J. Hall ${ }^{5,6}$, Tieying Shi Yu-Tao Xiang ${ }^{8,9,10}$ and Yanqing Tang $\mathbb{B}^{1,2}$

\begin{abstract}
Poor psychiatric status and sleep quality were common among frontline healthcare workers (FHWs) during the outbreak of the 2019 novel coronavirus disease (COVID-19), but the change in these mental health outcomes overtime remained unknown. This study compared the psychiatric status and sleep quality of FHWs during and after the COVID19 outbreak in China. FHWs who volunteered to work in Hubei province (the COVID-19 epicenter) were assessed at baseline during the COVID-19 outbreak and re-assessed when they returned to their place of origin (Liaoning province) after the COVID-19 outbreak. Participants' psychiatric status and sleep quality were measured with the Symptom CheckList-90 (SCL-90) and the Pittsburgh Sleep Quality Index (PSQI), respectively. A total of 494 FHWs was assessed at baseline and 462 at follow-up assessments. The prevalence of poor psychiatric status was $10.5 \%$ at baseline and increased to $14.9 \%$ at the follow-up assessment $(P=0.04)$. The corresponding figures of poor sleep quality at baseline and follow-up assessment were $16.4 \%$ and $27.9 \%$, respectively $(P<0.001)$. Multiple logistic regression analysis found that severe fatigue $(p=0.003, \mathrm{OR}=1.266,95 \% \mathrm{Cl}=1.081-1.483)$, poor sleep quality $(p<0.001, \mathrm{OR}=1.283,95 \%$ $\mathrm{Cl}=1.171-1.405)$, and history of pre-existing psychiatric disorders $(p<0.001, \mathrm{OR}=5.085,95 \% \mathrm{Cl}=2.144-12.06)$ were independently associated with higher odds of poor psychiatric status among the FHWs. Poor psychiatric status and sleep quality were common among FHWs during the COVID-19 outbreak, and the prevalence increased following their volunteer experiences. This suggests a critical need for longer-term psychological support for this subpopulation.
\end{abstract}

\section{Introduction}

The 2019 novel coronavirus disease (COVID-19) was first reported in late 2019 in Wuhan, Hubei Province, China ${ }^{1}$, and was declared by the World Health Organization as a pandemic in March $2020^{2}$. As of 6 November 2020, there have been 86,184 confirmed COVID-19 cases

\footnotetext{
Correspondence: Yu-Tao Xiang (xyutly@gmail.com) or

Yanqing Tang (tangyanqing@cmu.edu.cn)

${ }^{1}$ Department of Psychiatry, The First Affiliated Hospital of China Medical

University, Shenyang, Liaoning Province, China

${ }^{2}$ Department of Geriatric Medicine, The First Affiliated Hospital of China

Medical University, Shenyang, Liaoning Province, China

Full list of author information is available at the end of the article

These authors contributed equally: Yifang Zhou, Hailong Ding
}

in China, mostly originated in Hubei Province (the epicenter) $)^{3}$. To relieve the shortage of health professionals and control the disease transmission, over 42,600 healthcare workers were sourced from all parts of China and volunteered to combat the COVID-19 outbreak in Hubei Province ${ }^{4,5}$. The overwhelming clinical workload, fear of contagion, utmost concern about disease transmission, and perceived loneliness in isolation wards were associated with psychiatric problems among frontline healthcare workers (FHWs), particularly in the early stage of the COVID-19 outbreak $^{6-8}$. An online survey found that the prevalence of depression, anxiety, insomnia, and distress symptoms was $50.7 \%, 44.7 \%, 36.1 \%$, and

\section{(c) The Author(s) 2021}

(c) (i) Open Access This article is licensed under a Creative Commons Attribution 4.0 International License, which permits use, sharing, adaptation, distribution and reproduction c. in any medium or format, as long as you give appropriate credit to the original author(s) and the source, provide a link to the Creative Commons license, and indicate if changes were made. The images or other third party material in this article are included in the article's Creative Commons license, unless indicated otherwise in a credit line to the material. If material is not included in the article's Creative Commons license and your intended use is not permitted by statutory regulation or exceeds the permitted use, you will need to obtain permission directly from the copyright holder. To view a copy of this license, visit http://creativecommons.org/licenses/by/4.0/. 
73.4\%, respectively, among FHWs in China ${ }^{9}$. Commonly reported correlates of psychiatric problems included age, gender, having organic diseases, and pre-existing depression or anxiety ${ }^{10-14}$.

To lower the risk of psychiatric problems among FHWs, health authorities, psychiatric hospitals, and academic institutions and societies in China rapidly developed a range of responses including treatment guidelines and expert consensus ${ }^{15,16}$, online education ${ }^{17}, 24 \mathrm{~h}$ hotline services, online counseling, on-site crisis psychological interventions, and relevant research ${ }^{18}$. In addition, financial subsidy and social support were provided to FHWs, along with ensuring their safe working environment. Consequently, the prevalence of psychiatric problems among FHWs gradually reduced in subsequent studies. For instance, a survey conducted in the late stage of the COVID-19 outbreak found that the prevalence of poor mental health status, significant stress, and poor sleep quality was $10.8 \%, 23.2 \%$, and $18.9 \%$, respectively, among FHWs in Hubei province ${ }^{19}$.

Previous infectious disease epidemics, such as Severe Acute Respiratory Syndrome (SARS) and Ebola, were associated with increased risk of psychiatric problems during the outbreak ${ }^{20-23}$ and persistent psychiatric comorbidities among the FHWs following the outbreak. For example, symptoms of depression, anxiety, burnout, psychological distress, and posttraumatic stress were still common among FHWS within 1-3 years after the SARS outbreak $^{20,24,25}$.

After the large-scale COVID-19 outbreak had been well controlled in April 2020, several small-scale COVID-19 outbreaks occurred in some areas in China, such as Heilongjiang, Beijing, Qingdao, and Xinjiang, caused by imported cases from overseas. As the COVID-19 epidemic has been well controlled in China, most FHWs who volunteered to combat the COVID-19 outbreak in Hubei province have returned to their hometowns. To date, no longitudinal studies have examined the change of FHW's mental health status in China, which gave us the impetus to compare the mental health status of FHWs during and after COVID-19 outbreak. It is of paramount importance to develop effective preventive measures and offer timely treatment to reduce the long-term negative outcomes brought on by COVID-19. Findings emerging from this longitudinal study can be used to advise public health policy-makers how to best distribute limited mental health resources effectively.

\section{Participants and methods \\ Study design and participants}

This study was conducted between 21 February and 6 March 2020 (baseline assessment) during the COVID-19 outbreak in Hubei Province. Consecutive sampling was used. Due to the risk of transmission, traditional face-to-face interviews were not conducted; therefore, online surveys have been widely adopted ${ }^{6,9,26-29}$. In this study, a smartphone-based Wechat (a social media platform used by more than one billion people in China) Mini Program named PROP psychological CT system was used to collect the data. After scanning a Quick Response code, participants could complete the assessment using their smartphones. Inclusion criteria were as follows: (1) adults aged 18 years or above; (2) FHWs who had worked in the emergency medical assistance team from Liaoning to Hubei Province, to care for confirmed and suspected cases of COVID-19; and (3) participants who were able to understand the content of the assessment and provide written informed consent. All participants were re-assessed between 22 March and 14 April 2020 (follow-up assessment) using the same battery of instruments when they returned to their hometowns in Liaoning Province and during their mandatory 2-week centralized quarantine. The study protocol was approved by the Institutional Review Board of the First Affiliated Hospital of China Medical University.

\section{Measurements and data collection}

A data collection form was used to collect participants' basic socio-demographic and clinical characteristics, such as gender, age, educational level, marital status, parental status ("yes/no"), history of pre-existing psychiatric disorders ("yes/no"), occupation, work experience, working status, and good family support ("yes/no"). Participants' working status was assessed by the following standardized "yes/no" questions as follows: (1) "Have you ever directly cared for COVID-19 patients?"; and (2) "Are you familiar with the crisis response protocols and relevant knowledge?".

Psychiatric status was evaluated by the validated Chinese version of the Symptom CheckList-90 (SCL-90), which is a self-administered scale ${ }^{30,31}$ consisting of 90 items in 9 domains: somatization, obsessivecompulsiveness, interpersonal sensitivity, depression, anxiety, hostility, phobic anxiety, paranoid ideation, and psychoticism. Each item scored from 1 to 5 and the total score ranges from 90 to 450 . Higher scores indicated poorer mental health status. A SCL-90 total score of $\geq 160$ was considered as having "poor psychiatric status" 32 . Fatigue was measured using the numeric rating scale (NRS), ranging from " 0 " (no suffering from fatigue) to " 10 " (unbearably suffering from fatigue) ${ }^{33}$. A higher score indicates more severe fatigue and the score of $\geq 4$ was considered "clinically relevant fatigue" ("having fatigue" hereafter $)^{34}$. Sleep quality was evaluated by the Pittsburgh Sleep Quality Index (PSQI)-Chinese version ${ }^{35}$. The PSQI total score ranges from 0 to 21 , with the score of $>7$ considered as "having poor sleep quality"36. The Cronbach's $\alpha$ was above 0.69 for SCL-90 subscores ${ }^{37}, 0.92$ for the NRS on fatigue ${ }^{38}$, and 0.84 for PSQI ${ }^{36}$. 


\section{Data analysis}

The SPSS program Version 22.0 was used to analyze data. Normal distribution of continuous variables was evaluated using the Kolmogorow-Smirnov test. Basic demographic and clinical characteristics of FHWs between baseline and follow-up assessments, and between good and poor psychiatric status at follow-up were compared using $X^{2}$-tests for categorical variables, twoindependent samples $t$-tests for normally distributed continuous variables, and Mann-Whitney $U$-tests for skewed continuous variables. The independent associations between poor mental health and demographic and clinical characteristics at follow-up were examined using multiple logistic regression analysis with the "enter" method. Socio-demographic and clinical variables with significant group difference in univariate analysis were entered as independent variables. Significant level was set as $p<0.05$ for all tests (two-sided).

\section{Results}

Of the 2054 FHWs who volunteered in Hubei Province from Liaoning Province, 521 were consecutively invited to participate in this study at baseline and 494 completed the assessment, yielding a participation rate of $94.8 \%$ (494/ 521). Of them, 462 completed the follow-up assessment. Supplementary Table 1 showed the comparisons of participants' demographic and clinical characteristics between baseline and follow-up assessments. Basic demographic and clinical characteristics by psychiatric status at follow-up assessment are shown in Table 1. Kolmogorow-Smirnov tests revealed that PSQI and fatigue total scores were skewed.

Overall psychiatric status with the domain scores and poor sleep quality of participates were compared between baseline and follow-up assessments (Table 2). Compared with the baseline assessment, the prevalence of poor mental health significantly increased at follow-up assessment $[10.5 \% \quad(95 \%$ confidence interval $(95 \% \mathrm{CI})$ $7.8-13.2 \%)$ at baseline vs. $14.9 \%(95 \%$ CI $11.7-18.2 \%)$ at follow-up; $p=0.04$ ]. The prevalence of poor sleep quality also significantly increased $[16.4 \%$ (95\% CI 13.1-19.8\%) at baseline vs. $27.9 \%$ (95\% CI $23.8-32 \%)$ at follow-up; $p<$ 0.001 .

Univariate analyses revealed that there were significant differences between good and poor psychiatric status in terms of knowledge about crisis response, family support, history of pre-existing psychiatric disorders, fatigue, and PSQI total score (Table 1). Poorer sleep quality (odds ratio $(\mathrm{OR})=1.350,95 \% \mathrm{CI}=1.211-1.506, P<0.001)$ and history of psychiatric disorders $(\mathrm{OR}=3.384,95 \% \mathrm{CI}=$ $1.218-9.401, P=0.019)$ were independently and positively associated with poor psychiatric status at baseline (Supplementary Table 2). Multiple logistic regression analysis revealed that participants who reported more severe fatigue $(\mathrm{OR}=1.266,95 \% \mathrm{CI}=1.081-1.483, P=$ $0.003)$, poorer sleep quality $(\mathrm{OR}=1.283,95 \% \mathrm{CI}=$ $1.171-1.405, P<0.001)$, and history of psychiatric disorders $(\mathrm{OR}=5.085,95 \% \mathrm{CI}=2.144-12.06, \quad P<0.001)$ were independently and positively associated with poor psychiatric status at follow-up (Table 3).

\section{Discussion}

To the best of our knowledge, this was the first longitudinal study that assessed psychiatric status and sleep quality of FHWs during and after the COVID-19 outbreak. Our results showed that the prevalence of poor psychiatric status and sleep quality among FHWs significantly increased after the COVID-19 outbreak. The psychiatric status was evaluated with the SCL-90, as this is a comprehensive instrument on general mental health in nine domains and has been widely used in clinical services and research ${ }^{11,39-41}$, with satisfactory psychometric properties in Chinese populations ${ }^{37}$.

The increased prevalence of poor psychiatric status and sleep quality among FHWs after the COVID-19 outbreak could be attributed to several reasons. First, FHWs may have delayed psychiatric reactions to overwhelming clinical workload. For instance, coupled with excessive fear of contagion, posttraumatic stress disorder (PTSD) may occur among FHWs after the COVID-19 outbreak ${ }^{24}$. PTSD patients with delayed onset usually develop full symptoms weeks and months after the event ${ }^{42}$. In this study, we speculated that once the FHWs finished their heavy clinical duty, their psychological defense mechanism may relax, allowing them to recall the traumatic images of their clinical work, which could worsen their psychiatric status. Second, when FHWs worked in Hubei Province, they focused on their clinical duties without any interference of other psychological pressure. However, once they returned to their original working environments, they resumed facing great family pressure, dealing with complex interpersonal/collegial relationships, and academic competition. All these stressors could elevate the risk of psychiatric problems. This assumption was affirmed in a previous study that showed around $30 \%$ of clinicians developed new-onset episodes of psychiatric disorders, such as depression, anxiety, and substance use, after the SARS outbreak ${ }^{43}$. Another possible reason for exacerbation of symptoms could be their experience of being quarantined, which is associated with increased psychiatric symptoms ${ }^{44}$.

In this study, poor sleep quality was positively associated with poor psychiatric status, which is consistent with previous findings ${ }^{45-47}$. Poor sleep quality was not only common in patients with psychiatric disorders, but was considered as a predictor of future occurrence of certain psychiatric disorders, such as depression and 
Table 1 Basic demographic and clinical characteristics of participants who completed the follow-up assessment.

\begin{tabular}{|c|c|c|c|c|c|c|c|}
\hline \multirow[t]{3}{*}{ Variables } & \multirow{2}{*}{\multicolumn{2}{|c|}{$\begin{array}{l}\begin{array}{l}\text { Good } \\
\text { psychiatric status }\end{array} \\
(n=393)\end{array}$}} & \multirow{2}{*}{\multicolumn{2}{|c|}{$\begin{array}{l}\begin{array}{l}\text { Poor } \\
\text { psychiatric status }\end{array} \\
(n=69)\end{array}$}} & \multirow{3}{*}{$\begin{array}{l}\text { Statistics } \\
x^{2}\end{array}$} & \multirow[b]{3}{*}{ df } & \multirow[b]{3}{*}{$P$} \\
\hline & & & & & & & \\
\hline & $n$ & $\%$ & $n$ & $\%$ & & & \\
\hline Female & 314 & 79.9 & 60 & 87.0 & 1.896 & 1 & 0.168 \\
\hline High education (university and above) & 385 & 98.0 & 69 & 100 & 1.429 & 1 & 0.487 \\
\hline Married & 295 & 75.1 & 51 & 73.9 & 0.001 & 1 & 0.969 \\
\hline Having children & 244 & 62.1 & 48 & 69.6 & 1.412 & 1 & 0.235 \\
\hline Nurse & 292 & 74.3 & 52 & 75.4 & 0.035 & 1 & 0.852 \\
\hline Working more than 5 years & 328 & 83.5 & 62 & 89.9 & 1.824 & 1 & 0.177 \\
\hline Working in Hubei $\geq 8$ weeks & 193 & 49.1 & 30 & 43.5 & 0.745 & 1 & 0.388 \\
\hline Caring for critical COVID-19 patients & 143 & 36.4 & 48 & 40.6 & 1.231 & 1 & 0.267 \\
\hline Familiar with crisis response knowledge & & & & & 6.638 & 2 & 0.036 \\
\hline Very familiar & 236 & 60.1 & 52 & 75.4 & & & \\
\hline Familiar & 121 & 30.8 & 15 & 21.7 & & & \\
\hline Not familiar & 36 & 9.2 & 2 & 2.9 & & & \\
\hline Good family support & 357 & 90.8 & 55 & 10.2 & 7.533 & 1 & 0.006 \\
\hline History of pre-existing psychiatric disorders & 16 & 4.1 & 16 & 23.2 & 33.275 & 1 & $<0.001$ \\
\hline & Mean & SD & Mean & SD & $T / Z$ & $d f$ & P \\
\hline Age (years) & 35.14 & 7.044 & 36.55 & 7.101 & 1.535 & 460 & 0.125 \\
\hline Fatigue total score & 3.04 & 2.268 & 4.88 & 1.867 & -8.188 & $-{ }^{a}$ & $<0.001$ \\
\hline PSQI total score & 4.85 & 3.028 & 8.72 & 3.635 & -6.121 & $-{ }^{a}$ & $<0.001$ \\
\hline
\end{tabular}

$d f$ degrees of freedom, PSQI Pittsburgh Sleep Quality Index.

a Mann-Whitney U-tests. Bolded values $<0.05$.

anxiety $^{48,49}$. Some researchers suggested that early recognition and treatment of poor sleep quality may lower the risk of future psychiatric disorders ${ }^{50}$. As expected, both fatigue and history of psychiatric disorders were significantly associated with higher risk of poor psychiatric status. Fatigue is a common symptom of certain psychiatric disorders, such as anxiety and depression $^{42}$, and was significantly associated with lower quality of life ${ }^{51}$. Appropriate psychosocial interventions, such as cognitive behavioral therapy ${ }^{52}$, could improve fatigue. High stress and related problems of FHWs with a history of psychiatric disorders may trigger their pre-existing disorders or worsen residual psychiatric symptoms, which is supported by previous findings ${ }^{27,53}$.

The strengths of this study included the longitudinal design and use of validated and widely used assessment tools on psychiatric status and sleep disturbance. However, some limitations should be acknowledged. First, only one follow-up assessment was conducted in this study and hence, the long-term impact of COVID-19 outbreak on FHWs' psychiatric status and sleep quality could not be ascertained. Second, due to logistical reasons, only standardized scales were administered to evaluate FHW's psychiatric status, rather than using diagnostic instruments. In addition, some factors related to psychiatric status of FHWs, such as the length of stay in Hubei at the baseline assessment, were not recorded. Finally, FHWs in only one province (Liaoning Province) was recruited in this study, which may limit the generalizability of the findings to other regions of China.

In conclusion, poor psychiatric status and sleep quality were common among FHWs during the COVID-19 outbreak, and the prevalence of these conditions may increase after the COVID-19 outbreak. Long-term sustainable psychological support should be provided for this subpopulation. Prospective studies with longer follow-up period should be conducted to examine the long-term impact of the COVID-19 outbreak on FHWs' psychiatric status and sleep quality. 
Table 2 Comparisons of the SCL-90 total and domain scores between the assessments in Hubei and Liaoning Provinces.

\begin{tabular}{|c|c|c|c|c|c|}
\hline & \multirow{2}{*}{$\begin{array}{l}\text { Baseline (n} \\
=494) \\
N(\%)\end{array}$} & \multirow{2}{*}{$\begin{array}{l}\text { Follow-up } \\
(n=462) \\
N(\%)\end{array}$} & \multicolumn{3}{|c|}{ Statistics } \\
\hline & & & $x^{2}$ & df & $P$ \\
\hline $\begin{array}{l}\text { SCL-90 total score } \\
(\geq 160)\end{array}$ & $52(10.5)$ & $69(14.9)$ & 4.197 & 1 & 0.04 \\
\hline Somatization & $46(9.3)$ & $52(11.3)$ & 0.98 & 1 & 0.322 \\
\hline Obsession & 90 (18.2) & $101(21.9)$ & 1.982 & 1 & 0.159 \\
\hline $\begin{array}{l}\text { Interpersonal } \\
\text { relation }\end{array}$ & $44(8.9)$ & $56(12.1)$ & 2.634 & 1 & 0.105 \\
\hline Depressed & $44(8.9)$ & $58(12.6)$ & 3.332 & 1 & 0.068 \\
\hline Anxious & $37(7.5)$ & $43(9.3)$ & 1.028 & 1 & 0.311 \\
\hline Hostility & $32(6.5)$ & $35(7.6)$ & 0.442 & 1 & 0.506 \\
\hline Terrifying & $86(17.4)$ & $93(20.1)$ & 0.297 & 1 & 0.586 \\
\hline Bigoted & $18(3.6)$ & $36(7.8)$ & 7.709 & 1 & 0.005 \\
\hline $\begin{array}{l}\text { Mental } \\
\text { degeneration }\end{array}$ & $20(4)$ & $27(5.8)$ & 1.647 & 1 & 0.199 \\
\hline $\begin{array}{l}\text { Additional items } \\
\text { (diet/sleep) }\end{array}$ & $86(17.4)$ & $93(20.1)$ & 1.161 & 1 & 0.281 \\
\hline $\begin{array}{l}\text { PSQI total score } \\
(>7)\end{array}$ & $77(16.4)$ & $129(27.9)$ & 17.876 & 1 & $<0.001$ \\
\hline
\end{tabular}

df degrees of freedom, PSQI Pittsburgh Sleep Quality Index, SCL-90 Symptom CheckList-90.

Table 3 Factors independently associated with poor mental health at follow-up.

\begin{tabular}{|c|c|c|c|}
\hline \multirow[t]{2}{*}{ Variables } & \multicolumn{3}{|c|}{$\begin{array}{l}\text { Multivariate regression } \\
\text { analysis }\end{array}$} \\
\hline & $P$-value & OR & $95 \% \mathrm{Cl}$ \\
\hline Fatigue total score & 0.003 & 1.266 & $1.081-1.483$ \\
\hline PSQI total score & $<0.001$ & 1.283 & $1.171-1.405$ \\
\hline \multicolumn{4}{|l|}{ Crisis response knowledge } \\
\hline Not familiar & - & - & 1.0 \\
\hline Familiar & 0.226 & 2.797 & $0.529-14.777$ \\
\hline Very familiar & 0.059 & 4.616 & $0.941-22.645$ \\
\hline Good family support & 0.054 & 0.426 & $0.178-1.016$ \\
\hline $\begin{array}{l}\text { History of pre-existing psychiatric } \\
\text { disorders }\end{array}$ & $<0.001$ & 5.085 & $2.144-12.06$ \\
\hline
\end{tabular}

Cl confidential interval, OR odds ratio, PSQI Pittsburgh Sleep Quality Index. Bolded values $<0.05$.

\section{Acknowledgements}

The study was supported by the National Key R\&D Program of China (Grant \#2018YFC1311600 and 2016YFC1306900), the University of Macau (MYRG201900066-FHS), and Youth Backbone Support Program of China Medical University (QGZD2018033).

\section{Author details}

'Department of Psychiatry, The First Affiliated Hospital of China Medical University, Shenyang, Liaoning Province, China. ${ }^{2}$ Department of Geriatric Medicine, The First Affiliated Hospital of China Medical University, Shenyang, Liaoning Province, China. ${ }^{3}$ School of Public Health, China Medical University, Shenyang, Liaoning Province, China. ${ }^{4}$ School of Nursing, Hong Kong Polytechnic University, Hong Kong SAR, China. ${ }^{5}$ New York University (Shanghai), Shanghai, China. ${ }^{6}$ School of Global Public Health, New York University, New York, NY, USA. ${ }^{7}$ Department of Nursing, The First Affiliated Hospital of Dalian Medical University, Dalian, Liaoning, China. ${ }^{8}$ Unit of Psychiatry, Department of Public Health and Medicinal Administration, \& Institute of Translational Medicine, Faculty of Health Sciences, University of Macau, Macao SAR, China. ${ }^{9}$ Centre for Cognitive and Brain Sciences, University of Macau, Macao SAR, China. ${ }^{10}$ Institute of Advanced Studies in Humanities and Social Sciences, University of Macau, Macao SAR, China

\section{Conflict of interest}

The authors declare no competing interests.

\section{Publisher's note}

Springer Nature remains neutral with regard to jurisdictional claims in published maps and institutional affiliations.

Supplementary Information The online version contains supplementary material available at (https://doi.org/10.1038/s41398-020-01190-w).

Received: 28 July 2020 Revised: 1 December 2020 Accepted: 15 December 2020

Published online: 15 April 2021

\section{References}

1. Wang, C., Horby, P. W., Hayden, F. G. \& Gao, G. F. A novel coronavirus outbreak of global health concern. Lancet 395, 470-473 (2020).

2. World Health Organization. Coronavirus Disease 2019 (World Health Organization, 2020).

3. The State Council \& The People's Republic of China. Update on COVID-19. http://www.nhc.gov.cn/xcs/yqfkdt/202011/07daf90cf52d444d883c031305f3dc 84.shtml (2020).

4. The State Council \& The People's Republic of China. Press conference of the Joint Prevention and Control Mechanism of the State Council. http://www. gov.cn/xinwen/gwylflkjz48/index.htm (2020).

5. Xiang, Y.-T. et al. Tribute to health workers in China: a group of respectable population during the outbreak of the COVID-19. Int. J. Biol. Sci. 16, 1739-1740 (2020).

6. Lai, J. B. et al. Factors associated with mental health outcomes among health care workers exposed to coronavirus disease 2019. JAMA Netw. Open https:// doi.org/10.1001/jamanetworkopen.2020.3976 (2020).

7. Sockalingam, S., Clarkin, C., Serhal, E., Pereira, C. \& Crawford, A. Responding to health care professionals' mental health needs during COVID-19 through the rapid implementation of project ECHO. J. Contin. Educ. Health Prof. 40 211-214 (2020).

8. Calo, F., Russo, A., Camaioni, C., De Pascalis, S. \& Coppola, N. Burden, risk assessment, surveillance and management of SARS-CoV-2 infection in health workers: a scoping review. Infect. Dis. Poverty 9, 139 (2020).

9. Liu, N. et al. Prevalence and predictors of PTSS during COVID-19 outbreak in China hHardest-hit areas: gender differences matter. Psychiatry Res. 287, 112921 (2020).

10. Zhu, J. et al. Prevalence and influencing factors of anxiety and depression symptoms in the first-Lline medical staff fighting against COVID-19 in Gansu. Front. Psychiatry 11, 386 (2020). 
11. Zhang, W. et al. Mental health and psychosocial problems of medical health workers during the COVID-19 epidemic in China. Psychother. Psychosom. 89, 242-250 (2020).

12. Bohlken, J., Schomig, F., Lemke, M. R., Pumberger, M. \& Riedel-Heller, S. G. COVID-19 pandemic: stress experience of healthcare workers - a short current review. Psychiatr. Prax. 47, 190-197 (2020).

13. Zaharna, M. \& Guilleminault, C. Sleep, noise and health: review. Noise Health 12, 64-69 (2010).

14. Walker, M. P. The role of sleep in cognition and emotion. Ann. N. Y. Acad. Sci. 1156, 168-197 (2009).

15. General Office of the State Council of China. Key measures on improving the working conditions of frontline healthcare workers: caring for the physical and mental health of healthcare workers (in Chinese). http://www.gov.cn/ zhengce/content/2020-02/11/content_5477399.htm (2020).

16. Behavioral Medicine Branch of Chinese Medicine Association. Experts consensus on prevention and intervention of physical and mental exhaustion among frontline medical workers. https://www.cma.org.cn/art/2020/3/17/ art_2928_33681.html (2020).

17. Lin, L. \& Gaohua, W. COVID-19 National Mental Health Example Manual (Peking Univ. Medical Press, China, 2020).

18. Chen, Q. et al. Mental health care for medical staff in China during the COVID19 outbreak. Lancet Psychiatry 7, e15-e16 (2020).

19. Zhou, Y. et al. Tackling the mental health burden of frontline healthcare staff in the COVID-19 pandemic: China's experiences. Psychol. Med. https:/doi.org/ 10.1017/s0033291720001622 (2020).

20. McAlonan, G. M. et al. Immediate and sustained psychological impact of an emerging infectious disease outbreak on health care workers. Can. J. Psychiatry 52, 241-247 (2007).

21. Tam, C., Pang, E., Lam, L. \& Chiu, H. Severe acute respiratory syndrome (SARS) in Hong Kong in 2003: stress and psychological impact among frontline healthcare workers. Psychol. Med. 34, 1197-1204 (2004).

22. Grace, S., Hershenfield, K., Blackmore, E. \& Stewart, D. The occupational and psychosocial impact of SARS on academic physicians in three affected hospitals. Psychosomatics 46, 385-391 (2005).

23. Lehmann, M. et al. Ebola and psychological $s$ of health care professionals. Emerg. Infect. Dis. 21, 913 (2015).

24. Ping, $W$. et al. The psychological impact of the SARS epidemic on hospital employees in China: exposure, risk perception, and altruistic acceptance of risk. Can. J. Psychiatry 54, 302-311 (2009).

25. Maunder, R. et al. Long-term psychological and occupational effects of providing hospital healthcare during SARS outbreak. Emerg. Infect. Dis. 12, 1924-1932 (2006).

26. Wang, Y., Hu, Z., Feng, Y., Wilson, A. \& Chen, R. Changes in network centrality of psychopathology symptoms between the COVID-19 outbreak and after peak. Mol. Psychiatry https://doi.org/10.1038/s41380-020-00881-6 (2020).

27. Hao, F. Y. et al. Do psychiatric patients experience more psychiatric symptoms during COVID-19 pandemic and lockdown? A case-control study with service and research implications for immunopsychiatry. Brain Behav. Immun. 87, 100-106 (2020).

28. Zhou, S. J. et al. Prevalence and socio-demographic correlates of psychological health problems in Chinese adolescents during the outbreak of COVID-19. Eur. Child. Adolesc. Psychiatry 29, 749-758 (2020).

29. Yuan, B. et al. Correlation between immune response and self-reported depression during convalescence from COVID-19. Brain Behav. Immun. 88, 39-43 (2020).

30. Hua, J., Wenyuan, W. \& Mingyuan, Z. Zhong guo zheng chang ren SCL-90 ping ding jie guo de chu bu fen xi. Chin. J. Nerv. Ment. Dis. 12, 260-263 (1986).

31. Derogatis, L., Lipman, R. S. \& Covi, L. SCL-90: an outpatient psychiatric rating scale-preliminary report. Psychopharmacol. Bull. 9, 13-28 (1973).

32. Du, M.-L. et al. Assessment of mental health among nursing staff at different levels. Medicine 99, e19049 (2020).
33. Berger, A. M. et al. NCCN clinical practice guidelines cancer-related fatigue. J. Natl Compr. Canc. Netw. 8, 904-931 (2010).

34. Oldenmenger, W. H., de Raaf, P. J., de Klerk, C. \& van der Rijt, C. C. Cut points on 0-10 numeric rating scales for symptoms included in the Edmonton Symptom Assessment Scale in cancer patients: a systematic review. J. Pain. Symptom. Manag. 45, 1083-1093 (2013).

35. Buysse, D., Reynolds, C., Monk, T., Berman, S. \& Kupfer, D. The Pittsburgh Sleep Quality Index - a new instrument for psychiatric practice and rResearch. Psychiatry Res. 28, 193-213 (1989).

36. Liu, X. \& Tang, M. Q. Reliability and validity of the Pittsburgh Sleep Quality Index. Chin. J. Psychiatry 29, 29103-29107 (1996).

37. Shulin, C. \& Linjiang, L. Re-testing reliability, validity and norm applicatility of SCL -90. Chin. J. Neuropsychiatry 29, 5 (2003).

38. Wang, $X$. et al. Validation study of the Chinese version of the Brief Fatigue Inventory (BFI-C). J. Pain. Symptom. Manag. 27, 322-332 (2004).

39. Leichsenring, F. et al. To be or not to be improved: patients' perception of symptom improvement - linking the SCL-90-R to patient-rated global improvement in a large real-world treatment sample. Psychother. Psychosom. 89, 357-362 (2020).

40. Tian, F. et al. Psychological symptoms of ordinary Chinese citizens based on SCL-90 during the level I emergency response to COVID-19. Psychiatry Res. 288, 112992 (2020).

41. Vallejo, M., Jordán, C., Díaz, M., Comeche, M. \& Ortega, J. Psychological assessment via the internet: a reliability and validity study of online (vs paper-and-pencil) versions of the General Health Questionnaire-28 (GHQ-28) and the Symptoms Check-List-90-Revised (SCL-90-R). J. Med. Internet Res. 9, e2 (2007).

42. American Psychiatric Association. Diagnostic and Statistical Manual of Mental Disorders, Fifth Edition, DSM-5 (American Psychiatric Publishing, USA, 2013).

43. Lancee, W., Maunder, R. \& Goldbloom, D. Prevalence of psychiatric disorders among toronto hospital workers one to two years after the SARS outbreak. Psychiatr. Serv. 59, 91-95 (2008).

44. Gan, Y. et al. Immediate and delayed psychological effects of province-wide lockdown and personal quarantine during the COVID-19 outbreak in China. Psychol. Med. https://doi.org/10.1017/s0033291720003116 (2020).

45. Sanford, S., Bush, A., Stone, K., Lichstein, K. \& Aguillard, N. Psychometric evaluation of the beck anxiety inventory: a sample with sleep-disordered breathing. Behav. Sleep. Med. 6, 193-205 (2008).

46. Stein, M., Kroft, C. \& Walker, J. Sleep impairment in patients with social phobia. Psychiatry Res. 49, 251-256 (1994).

47. Gillin, J. Are sleep disturbances risk factors for anxiety, depressive and addictive disorders? Acta Psychiatr. Scand. Suppl. 393, 39-43 (1998).

48. Breslau, N., Roth, T., Rosenthal, L. \& Andreski, P. Sleep disturbance and psychiatric disorders: a longitudinal epidemiological study of young adults. Biol. Psychiatry 39, 411-418 (1996).

49. Neckelmann, D., Mykletun, A. \& Dahl, A. A. Chronic insomnia as a risk factor for developing anxiety and depression. Sleep 30, 873-880 (2007).

50. Ford, D. E. \& Kamerow, D. B. Epidemiologic study of sleep disturbances and psychiatric disorders. An opportunity for prevention? JAMA 262, 1479-1484 (1989).

51. Baek, Y., Jung, K., Kim, H. \& Lee, S. Association between fatigue, pain, digestive problems, and sleep disturbances and individuals' health-related quality of life: a nationwide survey in South Korea. Health Qual. Life Outcomes 18, 159 (2020).

52. Abrahams, H. J. G., Gielissen, M. F. M., Verhagen, C. \& Knoop, H. The relationship of fatigue in breast cancer survivors with quality of life and factors to address in psychological interventions: a systematic review. Clin. Psychol. Rev. 63, 1-11 (2018).

53. Vindegaard, N. \& Benros, M. E. COVID-19 pandemic and mental health consequences: systematic review of the current evidence. Brain Behav. Immun. 89 531-542 (2020) 